Journal for ImmunoTherapy of Cancer

\title{
Oncolytic adenovirus and gene therapy with EphA2-BiTE for the treatment of pediatric high-grade gliomas
}

\author{
Claudia Manuela Arnone, ${ }^{1}$ Vinicia Assunta Polito, ${ }^{1}$ Angela Mastronuzzi, ${ }^{1}$ \\ Andrea Carai, ${ }^{2}$ Francesca Camassei Diomedi, ${ }^{3}$ Laura Antonucci, ${ }^{1}$ \\ Lucia Lisa Petrilli, ${ }^{1}$ Maria Vinci, ${ }^{1}$ Francesco Ferrari, ${ }^{4,5}$ Elisa Salviato, ${ }^{4}$ \\ Marco Scarsella, ${ }^{6}$ Cristiano De Stefanis, ${ }^{6}$ Gerrit Weber (D) , ${ }^{1}$ \\ Concetta Quintarelli (D) , ${ }^{1}$ Biagio De Angelis, ${ }^{1}$ Malcolm K Brenner, ${ }^{7}$ \\ Stephen Gottschalk (i) , ${ }^{8}$ Valentina Hoyos (D) , ${ }^{7}$ Franco Locatelli, ${ }^{1,9}$ \\ Ignazio Caruana (D) , ${ }^{1}$ Francesca Del Bufalo (i) ${ }^{1}$
}

To cite: Arnone CM, Polito VA, Mastronuzzi A, et al. Oncolytic adenovirus and gene therapy with EphA2-BiTE for the treatment of pediatric highgrade gliomas. Journal for ImmunoTherapy of Cancer 2021;9:e001930. doi:10.1136/ jitc-2020-001930

- Additional supplemental material is published online only. To view, please visit the journal online (http://dx.doi.org/10. 1136/jitc-2020-001930).

IC and FDB contributed equally.

$\mathrm{IC}$ and FDB are joint senior authors.

Accepted 23 March 2021

Check for updates

(c) Author(s) (or their employer(s)) 2021. Re-use permitted under CC BY-NC. No commercial re-use. See rights and permissions. Published by BMJ.

For numbered affiliations see end of article.

Correspondence to Dr Francesca Del Bufalo; francesca.delbufalo@opbg.net

\section{ABSTRACT}

Background Pediatric high-grade gliomas (pHGGs) are among the most common and incurable malignant neoplasms of childhood. Despite aggressive, multimodal treatment, the outcome of children with high-grade gliomas has not significantly improved over the past decades, prompting the development of innovative approaches.

Methods To develop an effective treatment, we aimed at improving the suboptimal antitumor efficacy of oncolytic adenoviruses (OAs) by testing the combination with a gene-therapy approach using a bispecific T-cell engager (BiTE) directed towards the erythropoietin-producing human hepatocellular carcinoma A2 receptor (EphA2), conveyed by a replication-incompetent adenoviral vector (EphA2 adenovirus (EAd)). The combinatorial approach was tested in vitro, in vivo and thoroughly characterized at a molecular level.

Results After confirming the relevance of EphA2 as target in pHGGs, documenting a significant correlation with worse clinical outcome of the patients, we showed that the proposed strategy provides significant EphA2BiTE amplification and enhanced tumor cell apoptosis, on coculture with T cells. Moreover, T-cell activation through an agonistic anti-CD28 antibody further increased the activation/proliferation profiles and functional response against infected tumor cells, inducing eradication of highly resistant, primary $\mathrm{PHGG}$ cells. The gene-expression analysis of tumor cells and T cells, after coculture, revealed the importance of both EphA2-BiTE and costimulation in the proposed system. These in vitro observations translated into significant tumor control in vivo, in both subcutaneous and a more challenging orthotopic model. Conclusions The combination of OA and EphA2-BiTE gene therapy strongly enhances the antitumor activity of $O A$, inducing the eradication of highly resistant tumor cells, thus supporting the clinical translation of the approach.

\section{INTRODUCTION}

Along with embryonic tumors, high-grade gliomas (HGGs) are the most common malignant tumors of the brain in the pediatric age, encompassing glioblastoma (GBM), anaplastic astrocytoma and diffuse pontine glioma. ${ }^{1}$ Despite the progress in understanding the biology of pediatric highgrade glioma (pHGG) and the use of intensive, multimodal treatment approaches, including, more recently, targeted therapies, ${ }^{23}$ the outcome of these patients remains dismal, with a 5-year overall survival (OS) lower than $20 \%$ and invalidating long-term sequelae. Innovative, more effective and less toxic treatments are tremendously needed for these children.

In the past decades, immunotherapy proved to be a promising strategy for cancer treatment. ${ }^{45}$ A sophisticated form of immunotherapy is represented by oncolytic viruses: thanks to an increased knowledge of the molecular and biological characteristics of some pathogenic viruses and of the host immune response, it has become possible to engineer viruses in order to turn them into anticancer drugs. Adenoviruses (Ads)have been largely studied both as gene therapy vectors $^{6}$ to transfer genes in mammalian cells, or as oncolytic adenoviruses (OAs), genetically engineered to selectively replicate in and lyse tumor cells, sparing normal tissues. ${ }^{78}$ In preclinical studies, OA showed very promising results ${ }^{9-11}$; however, the clinical translation did not reach a comparable efficacy. Despite confirming the safety of the approach, ${ }^{12} 13$ only local and transient responses were demonstrated after intratumoral administration and results on intravenous infusion were largely unsatisfactory, ${ }^{14}$ highlighting the need for refinement of the approach. 
The combination of $\mathrm{OA}$ and gene therapy with an immunostimulatory gene, delivered by a second, replication-incompetent vector, represents a promising strategy. ${ }^{1516}$ We previously reported that the combination of OA with an incompetent Ad encoding for a suicide gene can significantly enhance the antitumor activity, maintaining the safety profile of the approach. ${ }^{17}$ We now propose to enhance the efficacy of OA through the combination with an Ad encoding for a bispecific T-cell engager (BiTE) $)^{18}{ }^{19}$ redirecting T cells against a selected tumor-associated antigen (TAA). BiTEs are chimeric proteins that combine the single-chain variable fragment domains (scFvs) of two different antibodies recognizing, respectively, an epitope on the CD3e of the T-cell receptor (TCR) complex and the selected TAA. The formation of an immunocytolytic synapse between $\mathrm{T}$ cells and tumor elements, via BiTEs, leads to the activation of the T cells and therefore promotes killing of the TAA-expressing tumor cell. ${ }^{2021}$ We selected to target the erythropoietinproducing human hepatocellular carcinoma A2 receptor (EphA2) TAA because a relevant correlation with tumor aggressiveness and worse patient outcome emerged in recent years in adult HGGs. ${ }^{22}{ }^{23}$ In this study, we demonstrate the feasibility and efficacy of combining OA with Ad-delivered gene therapy with an EpHA2-targeting BiTE (EphA2 adenovirus (EAd)); this approach is able to induce a potent tumor-specific cytotoxic activity leading to a long-lasting tumor control.

\section{MATERIALS AND METHODS Cloning}

The construction of the EphA2-BiTE cassette was previously described.$^{24}$ Specifically, the EphA2-specific engager consists of two scFvs (4H5 for EphA2 and OKT3 for the CD3) connected with a glycine (G) serine (S) linker ((G4S)3). This cassette was fused via a 2A-sequence to truncated human CD19 ( $\triangle$ CD19). Recombinant Ad 5F35 was constructed using the Adeno-X Expression System_1 from Clontech (Mountain View, California, USA).

\section{Virus production}

The OA (Ad5 $\Delta 24)$ was kindly provided by Dr Cerullo (University of Helsinki, Helsinki, Finland). Ad. $\Delta$ CD19 was produced by Baylor College of Medicine's Vector Development Lab following standard operating procedures (www.bcm.edu/vector).

\section{Cell culture}

Human GBM cancer cell lines U-373 MG and U87 were obtained from Merck (Darmstadt, Germany) and American Type Culture Collection, respectively. The cell lines were cultured following standard procedure, available in the online supplemental data.

\section{Adenoviral infection, transduction and coculture}

U373, U87 or pHGG cells were plated in a six-well tissue culture plates at $0.15 \times 10^{6}$ cells/well and after 24 hours were infected with OA 5 vp/cell or EAd $1000 \mathrm{vp/cell} \mathrm{or}$ the combination of both viruses as previously described. ${ }^{17}$ For the in vitro evaluation of the impact of the combined infection on T-cell activation, tumor cells were infected with OA $5 \mathrm{vp} /$ cell and EAd $250 \mathrm{vp} /$ cell and washed with phosphate-buffered saline (PBS) 72 hours after infection. For coculture, 24 hours after infection, effector cells were plated at the 10:1 effector:target (E:T) ratios. At day 5 of coculture, both residual tumor and $\mathrm{T}$ cells were assessed by flow cytometry (FACS) analysis.

\section{Quantitative real-time PCR (qRT-PCR) analysis}

Formalin-fixed pHGG samples were cut in ribbons of $40 \mu \mathrm{m}$ and processed to extract total RNA using the QIAmp RNeasy formalin-fixed, paraffin-embedded (FFPE) kit (QIAGEN, Hilden, Germany). Hundred $\eta \mathrm{g}$ of RNA were tested by one-step real-time PCR (RT-PCR) using the TaqMan probe for EphA2 (HS00171656_m1, Life Technologies, Carlsbad, California, USA). The difference in cycle threshold values of EphA2 was normalized to ACTB (HS01060665_g1, Life Technologies) and the fold change in expression was calculated relative to normal peripheral blood mononuclear cells, considered as negative controls using 12K quantitative PCR (qPCR) QuantStudio Flex Real-Time PCR system (Life Technologies). qPCR was also used to determine the gene expression of the E1A and the production of EphA2-BiTE. After 6 hours, 24 hours and 5 days, infected cells were collected and DNA extracted using QIAamp DNA mini kit (QIAGEN), according to the manufacturer's instructions. DNA samples were analyzed with specific primers (table 1) by SYBR Green technology (Life Technologies).

\section{Gene expression}

Cocultures of $\mathrm{T}$ lymphocytes and infected tumor cells were collected after 5 days. Tumor cells and T lymphocytes were then isolated via depletion strategy using anti-CD45 MicroBeads (Miltenyi, Bergisch Gladbach, Germany) to obtain tumor cells and anti-B7H3 PE (BD Bioscience), followed by anti-PE MicroBeads (Miltenyi) staining for the T-cell population. RNA was isolated using RNeasy Mini Kit (QIAGEN) and reversely transcribed with the cDNA SuperScript VILO cDNA Synthesis Kit (Life Technologies). Gene expression was then tested using TaqMan OpenArray Pathways Panels (Life Technologies) using 12K qPCR QuantStudio Flex Real-Time PCR system. Tumor cells were analyzed with the 'human cancer panel', while T cells were analyzed with 'human signal transduction and inflammation panels'.

\section{Xenograft mouse model for in vivo studies}

A detailed description of the mouse experiments is reported in online supplemental figure data.

\section{Statistical analysis}

Data are summarized as mean or median \pm SD or \pm SEM whenever indicated, depending on the characteristics of the analyzed samples. Student's t-test (two-sided) was used to determine statistically significant differences between 
samples, with a $\mathrm{p}$ value of $<0.05$ indicating a significant difference. When multiple comparison analyses were required, statistical significance was evaluated by a repeated measures analysis of variance (ANOVA) followed by a log-rank (Mantel-Cox) test. The patient survival data according to EphA2 expression were analyzed using R2 (http://r2.amc.nl) with a median cut-off modus.

For the RT-PCR-based expression profiles, we used the threshold cycle $(\mathrm{Ct})$ difference versus endogenous control and reference sample for normalization with inverted sign $(-\Delta \Delta \mathrm{Ct})$ as expression values (ie, the $\log 2$ transformation of RT-PCR relative expression quantification). For each gene in the gene-expression analysis, a linear regression model-accounting for sample variabilitywas used. P values for subgroups of therapy or presence/ absence of the costimulation molecule were estimated by one-way ANOVA test followed by Tukey's honestly significance difference. Family-wise error rate (FWER) was controlled at gene level. Only genes with at least three samples without NAs for each considered subgroup were retained, and the remaining missing values $(\sim 1.7 \%)$ were imputed (k nearest-neighbor algorithm)

The mouse survival data were analyzed using the KaplanMeier method and Fisher's exact test to measure statistically significant differences. Mice were matched based on the tumor signal for control and treatment groups before infusion. To compare the growth of tumors overtime, bioluminescence signal intensity was log transformed and compared using a t-test. We assumed a bioluminescence signal of $>10^{10}$ as an index of murine sacrifice. Graph generation and statistical analyses were performed using Prism V.4.0d software (GraphPad, La Jolla, California, USA). Significant $p$ values are shown in the graphs using the following reference: $*$ is used for $p$ values of $0.01-$ 0.05 ; ** for $\mathrm{p}$ values of $0.001-0.009$; $* * *$ for $\mathrm{p}$ values of $0.0001-0.0009$; and $* * * *$ for $p$ values of $<0.0001$.

\section{RESULTS}

\section{EphA2 is highly expressed in pediatric gliomas and is} associated with a worse patient outcome

To determine the relevance of the selected antigen EphA2 in HGG of the pediatric population, we evaluated its expression on primary pediatric glioma samples (WHO grading I-IV) obtained from our Pediatric Hematology-Oncology Department. The expression of EphA2 was tested first by immunohistochemistry (figure 1A) by an institutional expert neuropathologist. A clear correlation between the higher antigen expression and the grade of malignancy of the tumor was observed (figure 1A). To further validate our findings with a quantitative test, EphA2 antigen expression was analyzed by qRT-PCR, testing RNA extracted from pediatric gliomas samples, both low-grade glioma (LGG, WHO grading I-II) $(\mathrm{n}=27)$ and HGGs (WHO grading III-IV) (n=23) (figure 1B). This evaluation revealed an overall higher expression of the antigen in the HGG group as compared with the LGG group ( $\mathrm{p}=0.0039)$. Moreover, when we correlated the EphA2 expression with the progression-free survival (PFS) and OS of the patients, we observed a significant correlation between the high expression of the antigen and worse outcome of the patients $(\mathrm{p}=0.033$ and $\mathrm{p}=0.020$, $\mathrm{PFS}$ and OS, respectively) (figure 1C,D).

\section{Combination of $\mathrm{OA}$ and EAd significantly enhances the amplification of the transgene EphA2-BiTE}

One of the main advantages of the combinatorial approach relies on the ability of OA to provide the replication machinery needed to amplify the transgene vehiculated by the Ad, therefore exploiting the advantages of each single viral platform (figure $1 \mathrm{E}$ ). To validate this hypothesis, we first evaluated the replication of OA by infecting two commercial HGG lines (U373 and U87) with OA alone ( $5 \mathrm{vp} / \mathrm{cell})$, EAd alone (1000 vp/cell) or with both viruses (at the same vp/cell). At day 5 postinfection, an interval sufficient to allow the replication of OA, we analyzed the level of the E1A gene (carried only by the OA) by qRT-PCR in infected tumor cells. No difference was observed between the $\mathrm{OA}$ and the OA+EAd conditions, confirming that the coinfection does not impair the replication of the OA in any of the analyzed cell lines (figure $1 \mathrm{~F}$ ). We then evaluated the level of amplification of our EAd in all experimental conditions, analyzing the expression of the transgene EphA2-BiTE both by qRT-PCR, using specific pairs of primers (online supplemental table 1) (figure 1G), and by FC, measuring the level of the truncated CD19, reporter of the EphA2-BiTE (figure $1 \mathrm{H}$ ). Both techniques showed that the combined therapy is able to significantly amplify the expression of EphA2-BiTE, in both cell lines, as compared with the treatment with EAd alone.

OA+EAd enhances the antitumor response in longterm coculture with primary $\mathrm{T}$ cells, and further costimulation via $\alpha \mathrm{CD} 28$ is required to eradicate highly resistant tumor cell lines.

As shown in figure $1 \mathrm{E}$, the proposed combined approach aims at enhancing the antitumor response by the release of the product of the transgene, the EphA2BiTE, and the subsequent engagement and activation of $\mathrm{T}$ cells, thus inducing their cytotoxic activity at the tumor site. To prove this hypothesis, we infected the U373 and U87 cell lines with either OA ( $5 \mathrm{vp} /$ cell), EAd $(1000 \mathrm{vp} / \mathrm{cell})$ or OA+EAd. T cells isolated from healthy donors, were then cocultured with infected tumor cells at 10:1 E:T. After 4 days of coculture, tumor cells were analyzed for the expression of the apoptotic markers 7 -aminoactinomycin $\mathrm{D}$ and Annexin $\mathrm{V}$ by FC. As shown in figure 2A,B the presence of $\mathrm{T}$ cells significantly improves the antitumor response only in the presence of the EAd, in both the more sensitive cell line, U373, $(\mathrm{p} \leq 0.001)$ and in U87 $(p \leq 0.01)$, the latter showing a strong resistance to the oncolytic activity of the OA. Although promising, this effect is suboptimal, especially in the more resistant U87 cells, considering that the in vitro setting largely favors the infection and spread of $\mathrm{OA}$ and the interaction with $\mathrm{T}$ cells, underlining the need for further activation 
A

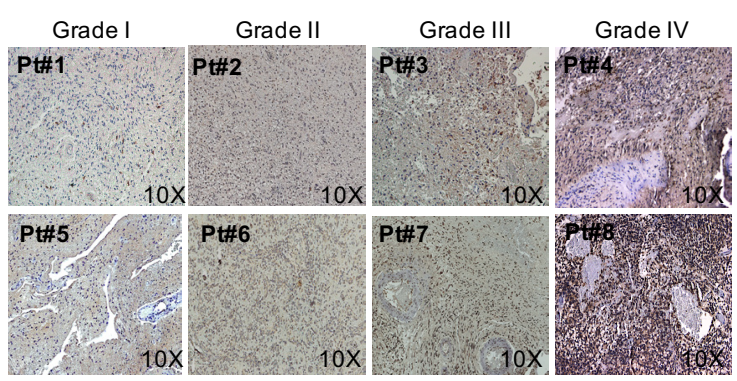

C

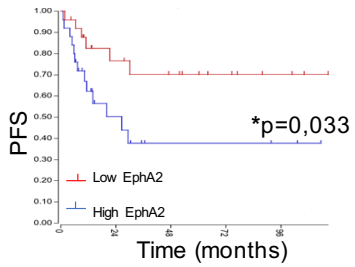

D

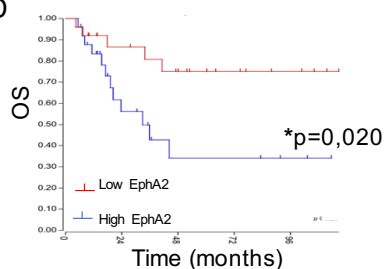

$\mathrm{F}$
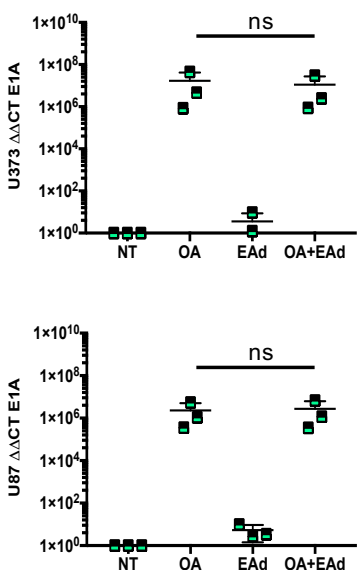

$E$

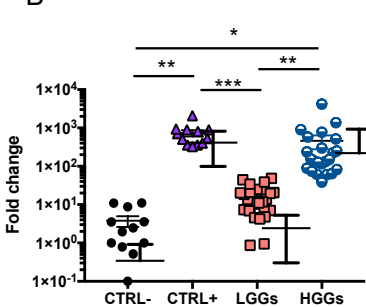

Figure 1 Relevance of EphA2 as pHGG target antigen and advantages of the OA+EAd combined therapy. (A) IHC expression in eight representative patients and (B) qPCR expression of EphA2 in the primary HGG and LGG samples, in healthy donor PBMC (Ctrl-) and in U373 (Ctrl+). (C) PFS and (D) OS estimates stratified for EphA2 RNA expression. (E) Schematic representation of the suggested experimental strategy; image created with BioRender. (F) Detection of E1A amplification by qPCR at day 5 after infection of U373 (upper panel, $n=3$ ) and U87 (lower panel, $n=3$ ) tumor cells with OA, EAd or with both viruses. (G) Quantification by qPCR of the expression of EphA2-BiTE at day 5 after infection and $(\mathrm{H})$ of the percentage of $\triangle$ CD19-positive tumor cells by FC, in U373 (upper panel) and U87 (lower panel). Data are expressed as mean \pm SD. Significant $p$ values are shown in the graphs using the following reference: * is used for $p$ values of $0.01-0.05$; ${ }^{* *}$ for $p$ values of $0.001-$ 0.009 ; ${ }^{\star * \star}$ for $p$ values of 0.0001-0.0009; and ${ }^{\star \star * \star}$ for $p$ values of $<0.0001$. BiTE, bispecific-T-cell engager; Ctrl, control; EAd, EphA2 adenovirus; EphA2, erythropoietin-producing human hepatocellular carcinoma A2 receptor; HGG, high-grade glioma; IHC, immunohistochemistry; LGG, low-grade glioma; ns, not significant; NT, untreated; OA, oncolytic adenovirus; OS, overall survival; PBMC, peripheral blood mononuclear cell; PFS, progression-free survival; pHGG, pediatric high-grade glioma; qPCR, quantitative PCR.

of the T-cell cytotoxic potential. Therefore, we included a costimulatory signal by adding a human monoclonal antibody (mAb) activating CD28, as a proof of concept (figure 2C), following the schema reported in figure 2D. By measuring the apoptotic signature through FC, we demonstrated that the addition of $\alpha \mathrm{CD} 28$ is able to 
A

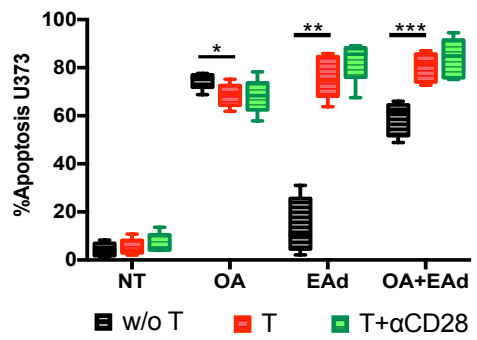

C

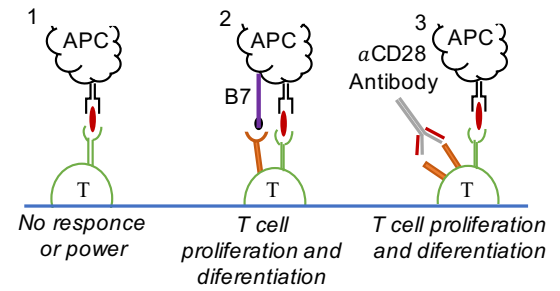

E

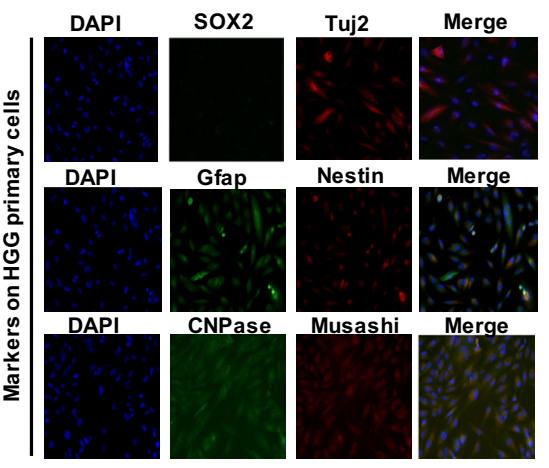

B

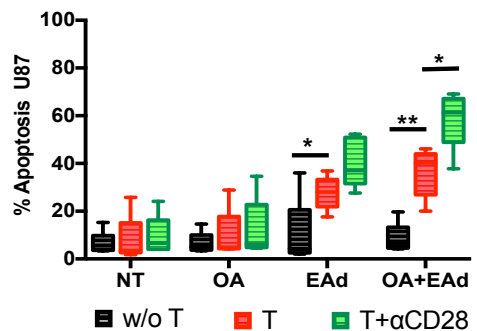

D
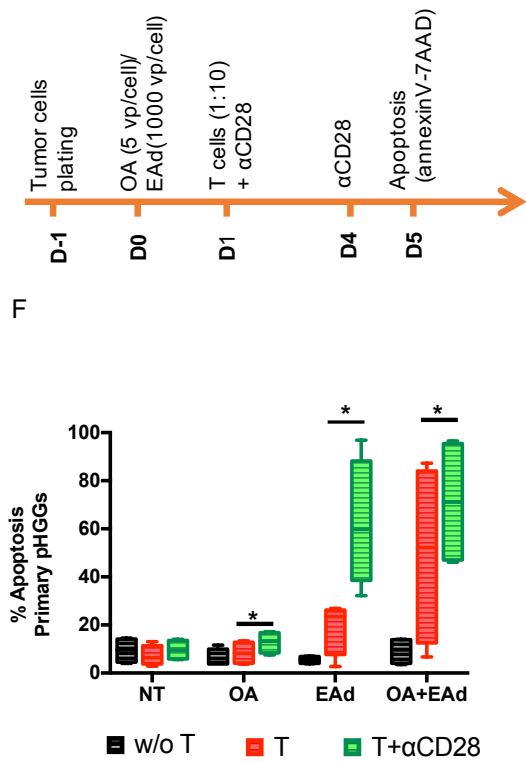

$\mathrm{H}$

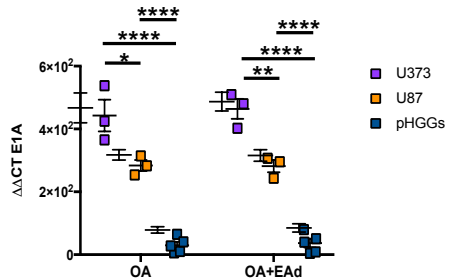

Figure 2 Improvement of the antitumor activity by OA+EAd in the presence of fully activated T cells and correlation between the expression of CAR and CD46 and the resistance to the treatment. (A) Percentage of apoptosis (7-AAD/Annexin V) analyzed by FC in U373 and (B) U87 cell lines. Data of T cells from 10 healthy donors are expressed as median \pm SEM. (C) Role of the CD28 costimulation on T cells. (D) Schematic representation of the experimental plan. (E) Characterization by immunofluorescence of one representative primary pHGG cell line. (F) Percentage of apoptosis (7-AAD/AnnexinV) analyzed by FC in primary pHGG cells. Data from fourcell lines are expressed as median \pm SEM. (G) Intensity of expression of CAR (left panel) and CD46 (right panel) on U373, U87 and pHGG cells. Data are expressed as median \pm SEM. (H) Evaluation of the expression of the E1A gene by qPCR 6 hours after infection of U373, U87 or pHGG cells. Data are expressed as median \pm SEM. 7-AAD, 7-aminoactinomycin D; APC, antigen presenting cells; CAR coxsackie-adenovirus receptor; DAPI, 4',6-diamidino-2phenylindole; EAd, EphA2 adenovirus; HGG, high-grade glioma; NT, untreated; OA, oncolytic adenovirus; pHGG, pediatric highgrade glioma; qPCR, quantitative PCR.

enhance T-cell activation and further improve the antitumor efficacy, significantly increasing the apoptosis of the more resistant U87 cells $(\mathrm{p}<0.01$, without vs with $\alpha \mathrm{CD} 28$ ) (figure 2B). Lastly, in order to further validate the efficacy of our strategy in vitro in a more representative tumor model of pHGG, we tested it on four different primary pHGG cell lines, established and characterized by immunofluorescence. The characterization showed a mild positivity for the staminal markers and a marked positivity of the oligondendrocytic and/or glial differentiation markers, confirming that a heterogeneous population of glioma tumor cells had been expanded (figure 2E). 
Using the same experimental plan described previously, we observed a strong resistance of primary pHGG cells to the OA (apoptosis: untreated (NT) $10.5 \% \pm 5.24 \%$, OA $4.45 \% \pm 1.43 \%$, without $\mathrm{T}$ cells). Four-day coculture with $\mathrm{T}$ cells, in the presence of EAd, was able to increase the tumor killing, which was further improved by the combination with OA (NT $6.40 \% \pm 3.81 \%$, OA $8.20 \% \pm 4.15 \%$, EAd $23.45 \% \pm 2.10 \%$ and OA+EAd $52.20 \% \pm 29.91 \%$ ). However, only the introduction of $\alpha \mathrm{CD} 28$ was able to significantly improve the antitumor effect of $\mathrm{T}$ cells as compared with the coculture in the absence of costimulation, ultimately inducing a relevant killing in the combined condition (EAd: $59.83 \% \pm 21.52 \%, \mathrm{p}=0023$; and OA+EAd: $72.65 \% \pm 23.99 \%, \mathrm{p}=0049$ ) (figure $2 \mathrm{~F}$ ).

In order to identify a potential mechanism responsible for the different response to the OA observed in the cell lines analyzed, we measured the expression of the coxsackie-adenovirus receptor (CAR) and of CD46 on U87, U373 and pHGG cells by flow cytometry. As shown in figure $2 \mathrm{G}$, the expression of both molecules was lower on U87 than on U373; pHGG cells as well show a lower expression than U373, although a relevant heterogeneity was observed. To confirm the hypothesis that the reduced expression of CAR can impact on the resistance to the $\mathrm{OA}$, we also evaluated the rate of infection of the three different cell lines by measuring the expression of the E1A gene by qRT-PCR 6 hours after infection (a timing which enables the full infection of the cells without allowing replication, therefore reflecting the level of infection). As shown in figure $2 \mathrm{H}$, U373 cells were infected by OA significantly more efficiently than U87 and pHGG. Moreover, to further confirm the reduced replication of OA and EAd in pHGG, we also analyzed the level of E1A and BiTE genes by qRT-PCR in infected tumor cells 5 days after infection. As shown in online supplemental figure 1 , the replication of both OA and EAd was lower than the most sensitive cell line U373 (figure 1F,G).

\section{Treatment with $0 \mathrm{~A}+\mathrm{EAd}$ induces memory phenotypes and an increase of activation markers of $T$ cells, further enhanced by aCD28}

To further characterize the T-cell response and its role in tumor eradication, we analyzed the phenotype of $\mathrm{T}$ cells after 4 days of coculture with tumor cells (either NT or infected with OA alone, EAd alone or the combination $\mathrm{OA}+\mathrm{EAd}$ ). A higher percentage of $\mathrm{CD}^{+}$than $\mathrm{CD} 8^{+} \mathrm{T}$ cells was detected in all the conditions, conserving the ratio detected in peripheral blood (online supplemental figure 2A,B). We then analyzed the memory phenotype and, in the coculture with the more sensitive U373 cells, we observed a modulation in the distribution of the naïve/memory subpopulations across the different conditions. In detail, EAd induces a significant reduction of the naïve population (NT vs EAd $\mathrm{p}=0.037$, OA vs EAd or $\mathrm{OA}+\mathrm{EAd} \mathrm{p}=0.015$ and 0.012 , respectively) and an increase of memory (central memory (CM) or effector memory (EM) ) subpopulations (figure 3A; left panel, black bars) . Furthermore, in the OA+EAd conditions, we observed a low percentage of terminally differentiated (ET) cells, suggesting the persistence of potential antitumor activity of the residual $\mathrm{T}$ cells $(\mathrm{p}=0.026)$ (figure $3 \mathrm{~A}$, left superior panel). The addition of the $\alpha \mathrm{CD} 28 \mathrm{mAb}$ did not modify this pattern and increased the effect observed (figure 3A, left inferior panel). A similar, although less significant, naïve/memory distribution was observed in the coculture with the more resistant U87 cells (figure 3A, right panels) and with primary pHGG cells (figure 3C).

We then further characterized the T-cell phenotype, analyzing the activation and exhaustion profiles. In the coculture with U373, EAd induces a significant increase of the activation and proliferation markers CD25 ( $\mathrm{p}=0.009)$, CD95 ( $\mathrm{p}=0.001)$ and Ki67 ( $\mathrm{p}=0.02)$ compared with $\mathrm{OA}$, further enhanced in the combined $\mathrm{OA}+\mathrm{EAd}$ condition (CD25, p=0.006; CD95, p=0.0001; and Ki67, $\mathrm{p}=0.03$ ) (figure 3B; left panels, dark gray bars). The exhaustion profile remains low in all the conditions, although a mild but significant increase is observed in the combined therapy $(\mathrm{p}<0.05)$ (figure 3B; left panels, dark gray bars). In the presence of $\alpha \mathrm{CD} 28$, although the overall level of exhausted cells remained low, the exhaustion profile increased significantly in the presence of EAd ( $\mathrm{OA}$ vs $\mathrm{EAd} / \mathrm{OA}+\mathrm{EAd}, \mathrm{p}=0.031$ and $\mathrm{p}=0.020$, respectively) (figure 3B; left panels, light gray bars). In coculture with U87 cells, the activating and proliferative potentials were driven mainly by EAd, without a significant contribution of the combination with OA (figure 3B; right panels, dark gray bars). Moreover, the addition of $\alpha \mathrm{CD} 28$ confirmed the same trend, although abrogating the statistical differences between the conditions (figure 3B; right panels, light gray bars). In order to evaluate in vitro the effect of the combinatorial treatment on the activation of the $\mathrm{T}$ cells, we next infected U87 with a reduced dose of EAd and included a washing step between the infection and the evaluation of the T-cell phenotype, reproducing a more challenging and realistic scenario. As shown in online supplemental figure $3 \mathrm{C}$, in the combinatorial approach, $\mathrm{T}$ cells had a higher expression of CD95 on both $\mathrm{CD}^{+}$and $\mathrm{CD} 8^{+}$cells, whereas $\mathrm{CD} 25$ expression was significantly more intense on the cytotoxic $\mathrm{CD}^{+}$population in the combined treatment than on infection with EAd only.

Lastly, in coculture with primary pHGGs cells, EAd increased significantly the expression of CD25 $(\mathrm{p}=0.01)$ and $\operatorname{CD} 95(\mathrm{p}=0.04)$ in the presence of $\alpha \mathrm{CD} 28$, as compared with $\mathrm{OA}$, this effect being further enhanced in the combined OA+EAd condition $(\mathrm{p}=0006)$ (figure 3D). Also, in this condition, we confirmed a mild, but significant, increase of the exhaustion profile.

Using the more sensitive U373 cell line, which revealed the most relevant differences between the experimental groups, and primary pHGG cells, we performed a deeper analysis, studying the contribution of both the $\mathrm{CD} 4$ and the CD8 subpopulations. As shown in online supplemental figure 2C,D, both populations recapitulated what had been observed in $\mathrm{CD}^{+}$cells in the naïe/memory and activation profiles. Moreover, both $\mathrm{CD}^{+}$and $\mathrm{CD} 8^{+}$ 
A
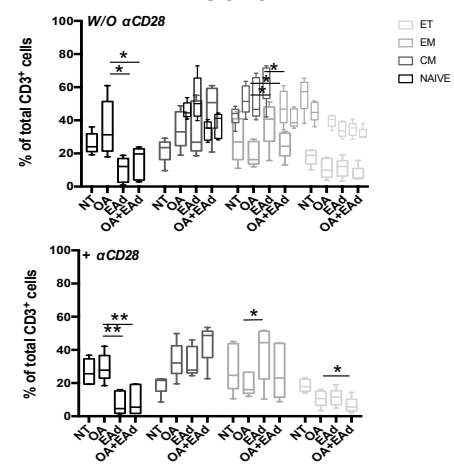

B
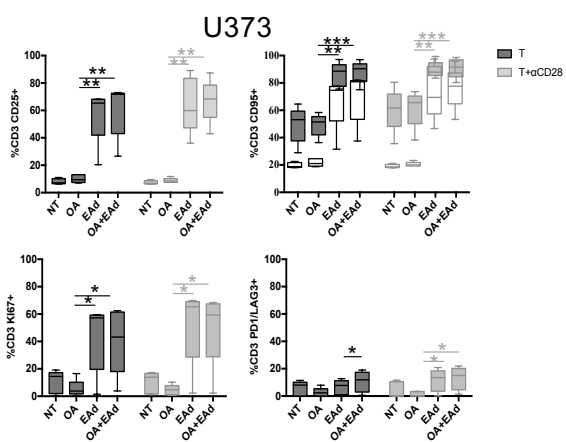

C
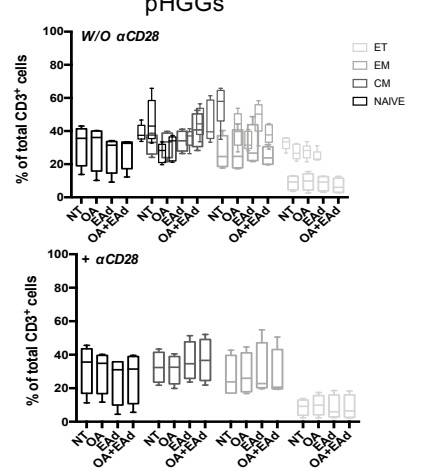

E

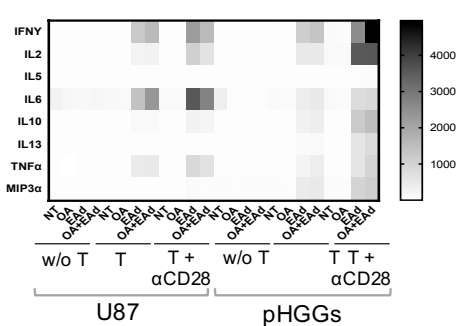

U87
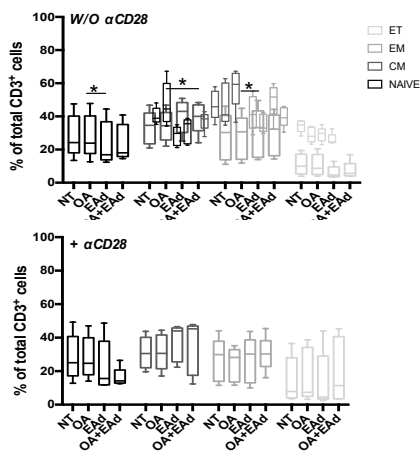

U87
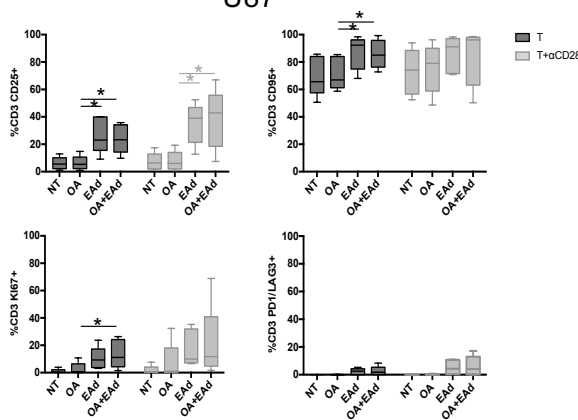

D

pHGGs
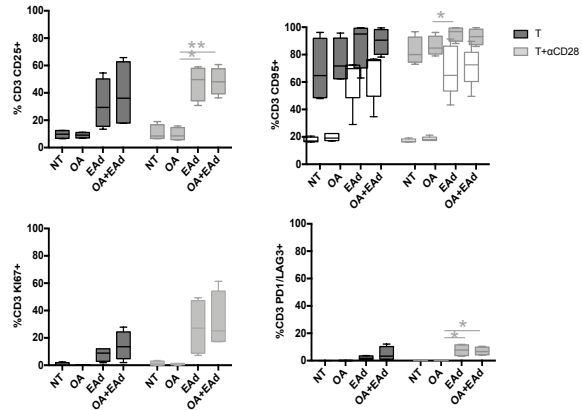

F

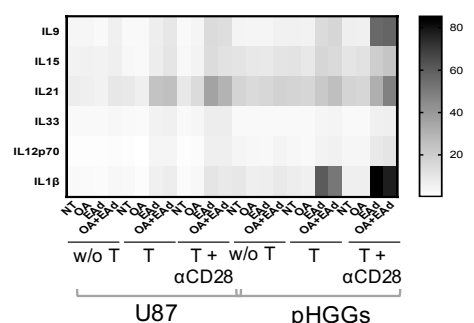

Figure 3 T-cell characterization after coculture with infected tumor cells. (A) T-cell naïve/memory distribution, expressed as percentage of total $\mathrm{CD}^{+}$cells and (B) activation/exhaustion profiles analyzed, by FC, after coculture with NT or infected (OA, EAd or OA+EAd) U373 (left panels) and U87 cell lines (right panels), without (upper graphs, A; dark gray bars, B) or with (lower graphs, A; light gray bars, B) $\alpha$ CD28. Data of T cells from 10 healthy donors are expressed as mean $\pm S E M$. (C) T-cell naïve/ memory distribution, expressed as percentage of total $\mathrm{CD}^{+}$cells, and (D) activation/exhaustion profiles, analyzed by FC, after coculture with NT or infected pHGG cell lines, without (upper graphs) or with (lower graphs) $\alpha$ CD28. Data from four donors are expressed as mean \pm SEM. (E,F) Cytokine release in supernatant after 24 hours of coculture with U87 or pHGG cell lines. Data are expressed as median \pm SEM. EAd, EphA2 adenovirus; NT, untreated; OA, oncolytic adenovirus.

T cells showd an increase of the expression of activation markers, on engagement with EAd, and recapitulated the significant contribution of $\alpha \mathrm{CD} 28$ (online supplemental figure $3 \mathrm{~A}, \mathrm{~B})$.
Functional activation of $T$ cells is driven by EAD, increases in the combined condition and is further enhanced by aCD28

In view of the milder activation and proliferation of T cells and the contribution of $\alpha \mathrm{CD} 28$ observed in the coculture 
with U87 and primary tumor cells, we further analyzed the functionality of $\mathrm{T}$ cells in these conditions by testing the Th1, Th2 and Th17 cytokines released in the supernatant 24 hours after coculture. As shown in figure 3E,F, we observed that EAd, either alone or in combination with $\mathrm{OA}$, drove the functional response of $\mathrm{T}$ cells in coculture with U87, with a higher release of proinflammatory cytokines, including interferon gamma (IFN- $\gamma$ ), interleukin (IL)-2, IL-6 and tumor necrosis factor alpha, as well as some anti-inflammatory cytokines, especially IL-10, compared with NT and OA alone (online supplemental table 2). The same trend was confirmed in the coculture with primary pHGG cells (online supplemental table 2). Lastly, the addition of $\alpha \mathrm{CD} 28$ significantly enhanced all the mentioned effects in both cocultures, with either U87 or pHGG (figure 3E,F).

\section{OA+EAd and $\alpha C D 28$ modulate several molecular pathways that sustain the antitumor activity mainly by enhancing T-cell activation and functionality}

In order to further investigate the impact of the treatment at a molecular level and identify the pathways involved in our approach, we performed a focalized gene-expression analysis both on tumor cells and on $\mathrm{T}$ lymphocytes after coculture and treatment, on the selection of each cell component.

On the tumor side, we clearly observed a clustering of the gene expression of NT and EAd-treated tumor cells on one side and of OA- and OA+EAd-treated conditions on the other side (figure 4A). However, further analysis of the modulation of the gene expression revealed a finer pattern, with a significantly wider modulation when comparing the combined infection versus the EAd or the NT conditions, as well as in the OA-infected tumor cells versus EAd or NT (figure 4B). When analyzed for the presence of $\mathrm{T}$ cells, the main driver of a significant gene modulation in tumor cells is the presence of lymphocytes, with or without $\alpha \mathrm{CD} 28$ (figure 4C). Therefore, we further characterized the peculiar sets of modulated genes in the tumor cells and the factors impacting on these modulations using the linear regression model coupled with ANOVA test. In detail, this analysis revealed the main role of EAd and $\alpha \mathrm{CD} 28$ in driving a powerful modulation of relevant pathways, with particular reference to the cellular response to stress and external stimuli, to the interleukin signaling as well as to the reduction of transforming growth factor beta signaling (figure $4 \mathrm{D}$, upper panel, and online supplemental figure 4). The combined infection, and even more in presence of $\alpha \mathrm{CD} 28$, has a strong impact on the modulation of genes of the pathways involved in the signaling by interleukins in tumor cells and in the organization of extracellular matrix (figure 4D, lower panel).

On T lymphocytes, the analysis underlined the role of EAd on the modulation of several genes involved in both signal transduction and inflammation (such as the overexpression of IFN- $\gamma$ and IL1R2 gene), this phenomenon being further implemented in the combined treatment with OA+EAd (figure 4E). The gene-ontology analysis and the deeper analysis of both upregulated and downregulated genes in $\mathrm{T}$ cells revealed how the combined treatment significantly modulates the expression of several relevant pathways in T cells, inducing, in particular: (1) an activation of the T-cell effector function and of Th1 cytokines, especially IFN- $\gamma$ (ie, upregulation of IFNG, FAS, and FASLG genes); (2) an overexpression of the gene of the costimulatory molecule CD86; (3) an increase in the production of chemokines capable of recruiting $\mathrm{T}$ cells and other immune cells at the tumor microenvironment (ie, CCL20, CXCL9, CCL1, and CCR1); (4) a reduction of the transcriptional regulation of the regulatory $\mathrm{T}$ cell differentiation by RUNX1, induced by OA and attenuated in the combined treatment; and (5) the activation of cell replication, as shown by the upregulation of genes of the cell cycle (mainly driven by EAd but amplified in the combination with $\mathrm{OA}$ ) (online supplemental figure $5 \mathrm{~A})$. Moreover, the global analysis on the contribution of $\mathrm{OA}$ and EAd interestingly revealed that the OA treatment is able to downregulate many genes involved in inflammation pathways, whereas the presence of EAd induces an upregulation of several relevant genes of the transduction and inflammatory pathways (online supplemental figure $5 \mathrm{~B})$. The administration of $\alpha \mathrm{CD} 28$ modulates the genes involved in transduction and inflammation with different impact in the different treatment conditions (figure $4 \mathrm{~F}, \mathrm{G}$ ). Indeed, the effect of $\alpha \mathrm{CD} 28$ depends on the presence of the BiTE, encoded by EAd and amplified in the combined condition, being essential in enhancing the modulation of the aforementioned genes and activated pathways induced by the engager (online supplemental figures 6 and $4 F, G$ ). Of note, the introduction in the experimental conditions of the costimulation is able to upregulate significantly the pathways of the immune system and of signaling by cytokines, strongly enhancing the counteracting effect of the EAd over the immunesuppressive effect shown by OA alone (online supplemental figure 6).

\section{OA+EAd induces a strong and persistent in vivo tumor control after infusion of $\mathrm{T}$ cells and $\alpha \mathrm{CD} 28$}

To validate the antitumor efficacy of our combinatorial approach OA+EAd in vivo, we established two different murine models, using female immunodeficient NSG mice: a subcutaneous and an orthotopic model. In both settings, a gene-modified U373 cell line expressing the Fire Fly Luciferase (FF.Luc), in frame with green fluorescent protein (GFP), was used to follow the tumor growth overtime by bioluminescence imaging. As shown in figure $5 \mathrm{~A}$, in the subcutaneous model, FF.Luc/GFPU373 cells were implanted in the right flank of the mouse. After engraftment, animals were treated intratumorally with either PBS, OA $\left(6 \times 10^{6} \mathrm{vp} / 100 \mu \mathrm{L}\right)$ or EAd $\left(6 \times 10^{7} / 100 \mu \mathrm{L}\right)$ or OA+EAd $\left(6 \times 10^{6} \mathrm{vp}+6 \times 10^{7} \mathrm{vp} / 100 \mu \mathrm{L}\right)$, respectively. Then, mice received an intravenous infusion of $\mathrm{T}$ cells, a second intratumoral infusion of viruses supplemented with $\alpha \mathrm{CD} 28$ followed by a further 
A
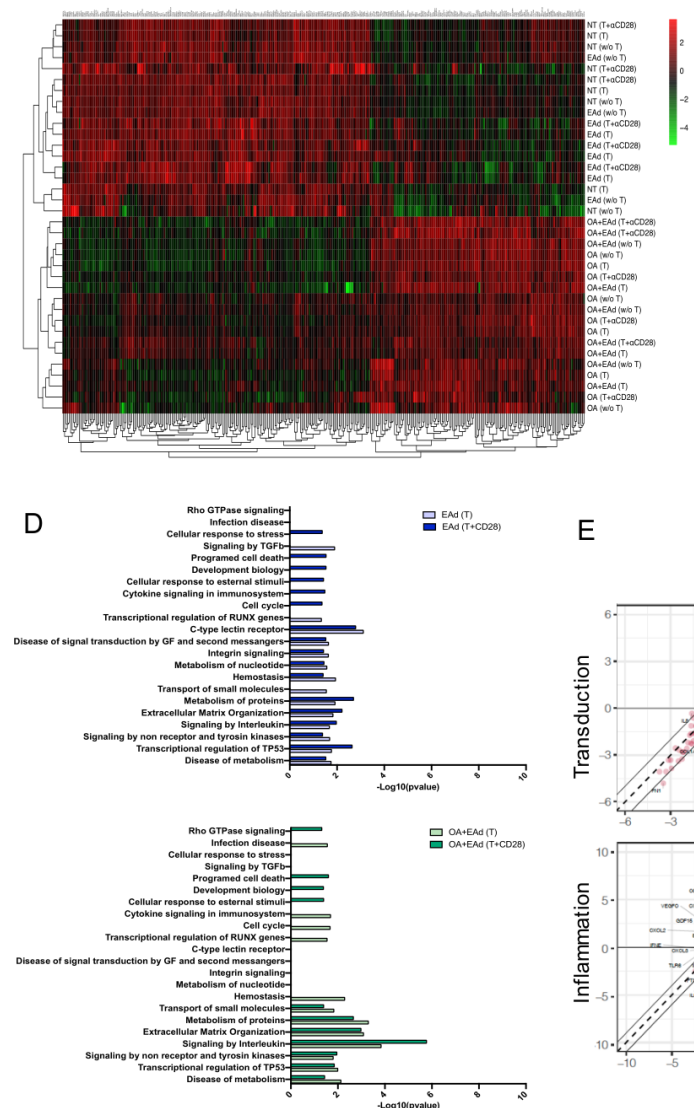

E

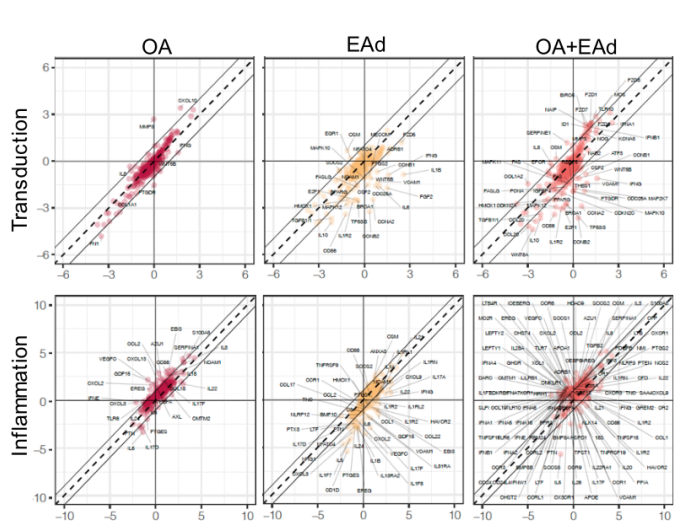

$\mathrm{F}$
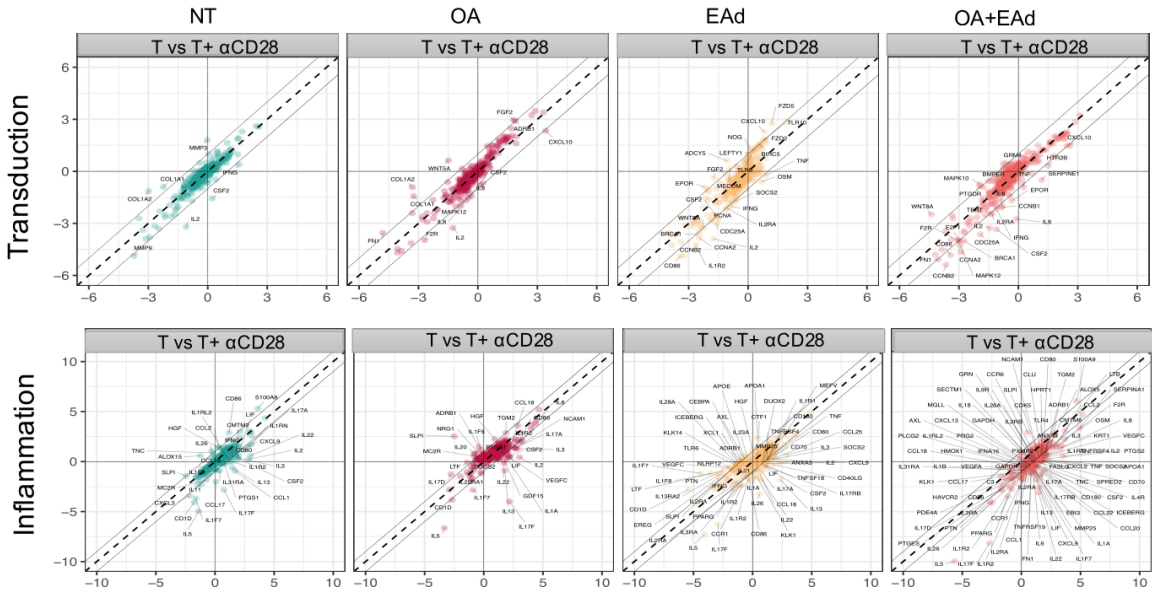

Figure 4 Gene-expression analysis on the contribution of the combined therapy, of T cells and of the $\alpha$ CD28 costimulation molecule. (A) Heatmap analysis of enriched genes involved in tumor pathways, on U87 tumor cells, either NT or after infection with either OA or EAD or OA+EAd, and in the presence or absence of the $\alpha \mathrm{CD} 28$ costimulation molecule; three biological replicates for each treatment condition are shown. Evaluation of the phenotypical variation based on differentially expressed enriched genes in tumor cells in the different treatment conditions (NT, OA, EAd and OA+EAd) (B) and T cells with or without $\alpha C D 28$ (C). Pathway enrichment based on gene ontology of enriched genes involved in tumor pathways in EAd+Tand

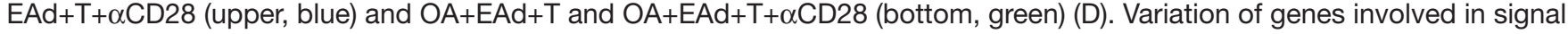
transduction (upper) and inflammation (bottom) on T lymphocytes after coculture with non-infected tumor cells (baseline) versus coculture with OA, EAd or OA+EAd-infected tumor cells (E) and the benefit of the addition of $\alpha \mathrm{CD} 28$ costimulation molecule in both transduction-related $(F)$ and inflammation-related $(G)$ genes. Dotted line shows the bisector, while the two parallel solid lines represent the halving or doubling of the expression. EAd, EphA2 adenovirus; NT, untreated; OA, oncolytic adenovirus.

$\alpha \mathrm{CD} 28$ intraperitoneal administration. Tumor growth was measured weekly with the Xenogen-IVIS Imaging
System. As shown in figure 5B,C and in online supplemental figure $7 \mathrm{~A}$, the combinatorial treatment $\mathrm{OA}+\mathrm{EAd}$ induces an earlier tumor control as compared with the 
A

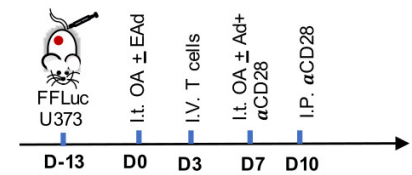

C

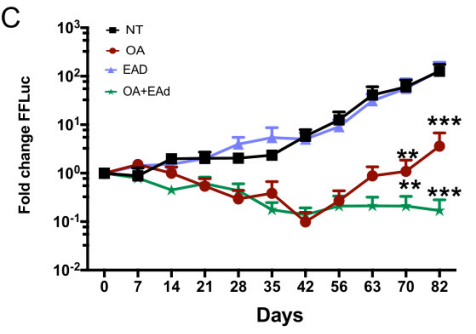

D

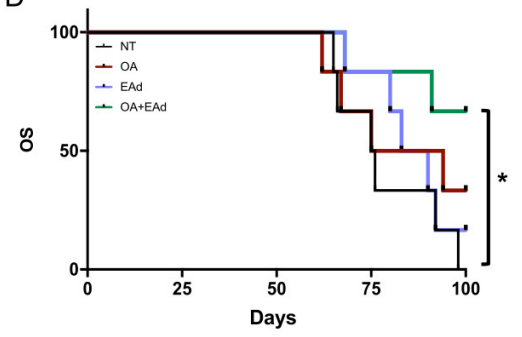

E

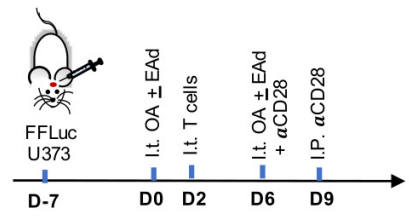

G

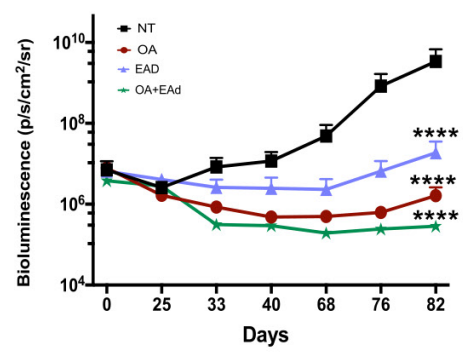

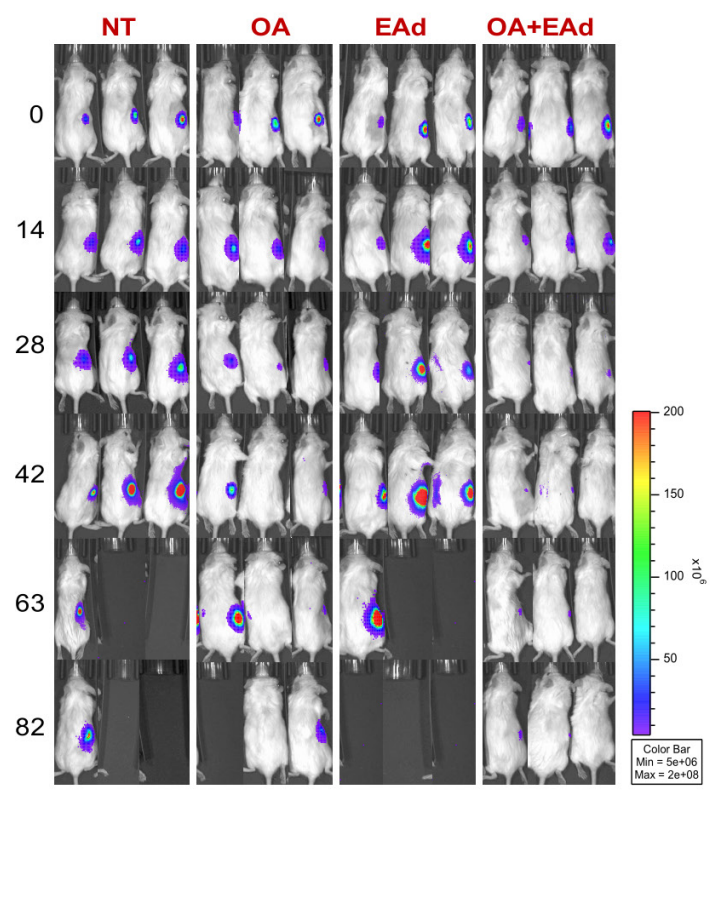

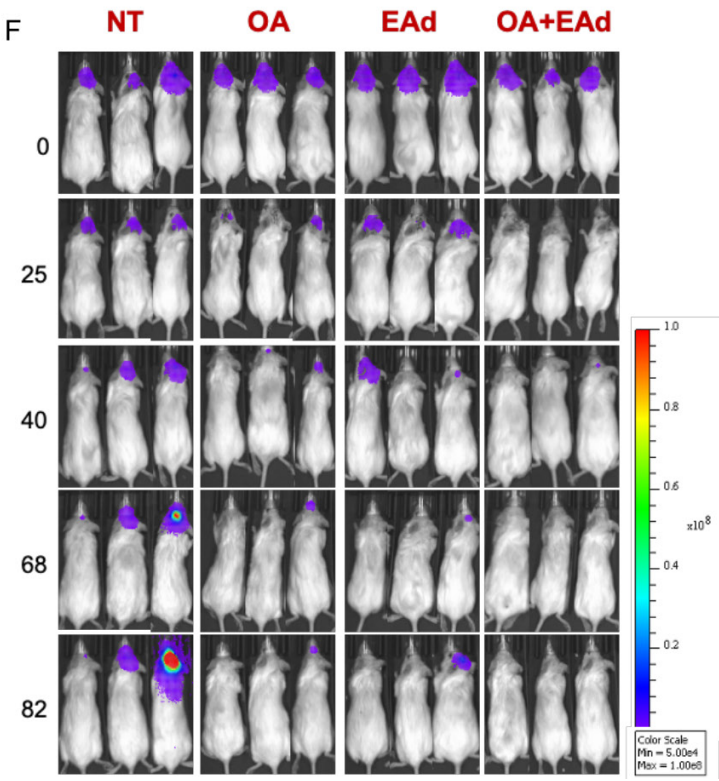

Figure 5 In vivo testing of the combinatorial approach in two mouse models of tumor engraftment, namely, subcutaneous and orthotopic. (A) Experimental design of subcutaneous mouse model; (B) bioluminescence signal of the engrafted tumors evaluated by in vivo imaging system over time; three representative mice per group are shown; (C) evaluation of the tumor growth over time, expressed as fold change of the bioluminescence signal of the four groups of treatment $(n=24)$. Data are summarized as mean \pm SEM. (D) Kaplan-Meier curve estimating the OS. (E) Experimental design of the orthotopic mouse model; $(F)$ bioluminescence signal of the engrafted tumors evaluated by IVIS imaging system over time; three representative mice per group are shown; $(G)$ evaluation of the tumor growth over time, measured through bioluminescence, of the four groups of treatment ( $n=6$ mice/treatment group). Data are summarized as mean \pm SEM. EAd, EphA2 adenovirus; NT, untreated; OA, oncolytic adenovirus; OS, overall survival.

other groups. Moreover, overall, a significantly improved tumor control was observed for the group that received OA+EAd $(p<0.0001)$, representing the only group with a persistent, long-term tumor reduction (figure 5C). We then evaluated the importance of the costimulation in the combined treatment by comparing the tumor control of this treatment group with or without administration of $\alpha \mathrm{CD} 28$. As shown in online supplemental figure 7C, in the absence of costimulation, the tumor growth is significantly less controlled than in the group of animals treated with the same viruses and $\alpha \mathrm{CD} 28$, as shown by the significantly lower reduction of bioluminescence signal. 
Overall, this stronger antitumor activity translates into a significantly increased OS for this group of treatment, as shown in figure $5 \mathrm{D}$.

To further characterize the in vivo antitumor activity in a more relevant tumor model, we developed an orthotopic HGG mouse model in which the FF.luc/GFP-U373 cells were stereotaxically implanted in the supratentorial brain region of the mice. Viruses were infused intratumorally 7 days later at the same concentration used in the previous model (figure 5E). Moreover, mice received $\mathrm{T}$ cells intratumorally, a second dose of the viruses in the presence of $\alpha \mathrm{CD} 28$ intratumorally and, lastly, a second dose of $\alpha \mathrm{CD} 28$ intraperitoneally. As shown in figure 5F,G, the bioluminescence reveals a significant tumor reduction with either EAd or OA or OA+EAd, as compared with NT $(p<0.0001)$, but the presence of OA was crucial to provide a more relevant tumor control in this model. Interestingly, the group that received the combined treatment $\mathrm{OA}+\mathrm{EAd}$ obtained an earlier and stronger tumor control (figure 5F,G and online supplemental figure 7B) and represented the only group maintaining the antitumor response at the latest time point of analysis, whereas the animals treated with either EAd or OA started to lose the response.

\section{Persistent T-cell activation, without any toxicity, is observed in vivo with the combination $\mathrm{OA}+\mathrm{EAd}$ and infusion of $\mathrm{T}$ cells and aCD28}

Having shown the efficacy of the combined treatment, we further characterized the in vivo response by analyzing the tumor tissues and the persistence and activation of the circulating human T cells.

The H\&E analysis of the brain of orthotopically engrafted mice confirmed the antitumorous effect induced by $\mathrm{OA}+\mathrm{EAd}$, showing absence or significantly smaller tumors, in the few mice still presenting a residual mass (in red, figure 6A). Moreover, the staining for the human Ki-67 shows highly proliferating residual tumors in the control mice, whereas very low to negative Ki-67 tumor cells were detected in the mice treated with the combined approach.

In the subcutaneous model, we analyzed the human $\mathrm{CD} 45^{+}$cells in the peripheral blood by bleeding at two different time points, namely, 40 days (when a relevant difference of tumor signal between the groups began to be evident) and 60 days after treatment. We observed that the T-cell expansion was initially comparable in the OA and $\mathrm{OA}+\mathrm{EAd}$ groups, probably driven by the recognition of the adenoviral antigens (figure 6B, left panel). However, the expansion persisted further only in the combinatorial approach and was still relevant at day 60 after infusion (figure 6C, left panel). These results correlated with the tumor control observed, that resulted superimposable between the OA and OA+EAd groups until day 40 and then significantly improved in the OA+EAd group. We then further characterized the expanded $T$ cells by analyzing the activation/exhaustion phenotype of the $\mathrm{CD}^{+}$and $\mathrm{CD}^{+}$subpopulations in the $\mathrm{OA}$ and $\mathrm{OA}+\mathrm{EAd}$ groups, by FC. We observed that, despite the expansion of circulating T cells after 40 days in the OA group, the activation of $\mathrm{CD} 4^{+}$and $\mathrm{CD} 8^{+}$subpopulations was reduced as compared with the combined OA+EAd group, and only a mild exhaustion profile was observed in both groups (figure 6B, right panel). Interestingly, the same trend was preserved at 60 days (figure $6 \mathrm{C}$, right panel). In view of the strong expansion, persistence and activation of $\mathrm{T}$ cells, we then aimed at characterizing the impact of the combined treatment on the recruitment and activation of $\mathrm{T}$ cells at the tumor site. Therefore, we sacrificed the animals 10 days after treatment and analyzed the tumorinfiltrating human lymphocytes. As show in figure $6 \mathrm{D}$, we could detect a relevant infiltration of human $T$ cells in the tumor mass only in the combined treatment. Notably, these infiltrated $\mathrm{T}$ lymphocytes displayed a strong activated phenotype with a prevalence of CM and EM cells for the $\mathrm{CD}^{+}$subpopulation and of $\mathrm{EM}$ for the $\mathrm{CD} 8^{+} \mathrm{T}$ cells (figure 6D).

Lastly, to validate the safety of our treatment, we first evaluated in vitro the potential toxicity of the approach by performing cocultures between infected primary normal cells and $\mathrm{T}$ cells, using the same condition tested with tumor cells. In details, we evaluated by flow cytometry the apoptosis of mesenchymal stromal cells for the mesenchymal tissues, CD $34^{+}$hematopoietic stem cells, the hematopoietic compartment, and primary normal brain tissue cells. As shown in online supplemental figure 8 , no significant increase in the apoptosis was detected against any primary normal cell.

Despite the lack of any sign of mice discomfort, we then analyzed in vivo the morphology of several tissues (brain, heart, intestine, liver, lung, kidney and bladder) for signs of cytopathy induced by the virus by $\mathrm{H} \& \mathrm{E}$. As shown in figure $6 \mathrm{D}$, no cellular or structural anomalies of the analyzed tissues in $\mathrm{OA}+\mathrm{EAd}$ were present, with the tissue histology being comparable to the NT condition.

\section{DISCUSSION}

In order to address the limitations observed in several clinical applications using OA, in this study, we developed a novel promising strategy to treat pHGG by combining $\mathrm{OA}$ and gene therapy introducing an immunostimulatory gene, EphA2-BiTE (EAd), delivered by a second replication-incompetent adenoviral vector. The recruitment and redirection of $\mathrm{T}$ cells toward the target antigen EphA2 and the induction of their cytotoxic activity reinforce the oncolysis provided by OA, restoring a strong proinflammatory microenvironment. This translates into a significantly better tumor control, thanks to the targeting of those tumors, or parts of the tumor, displaying resistance to the lytic activity of $\mathrm{OA}$. We also clearly show that the introduction of a costimulatory signal is essential in order to produce a significant and persistent tumor control, especially in more stringent models, such as primary pHGG cells. 
A

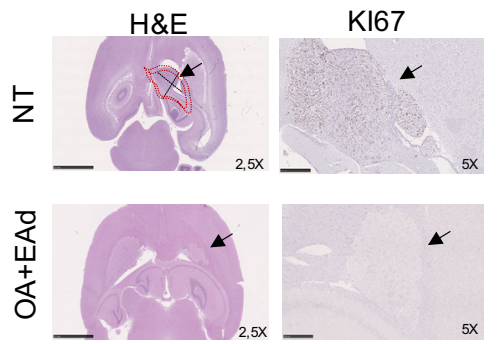

B

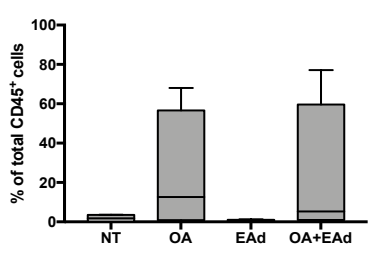

C

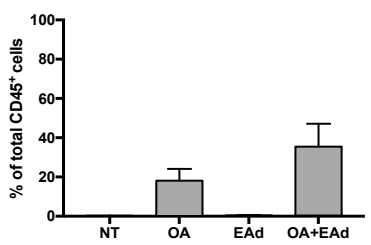

$H \& E$
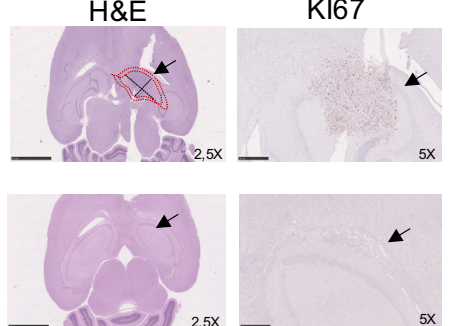

Day 40

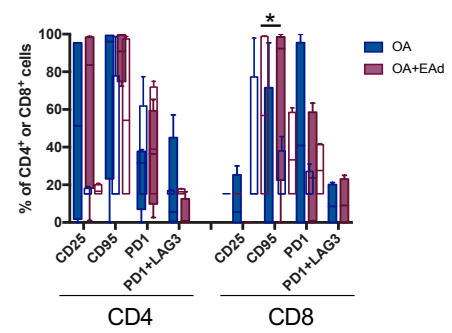

Day 60

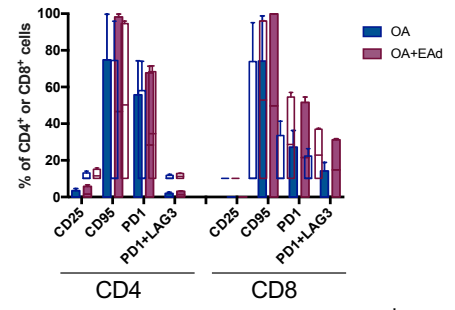

D

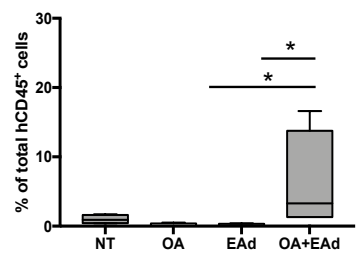

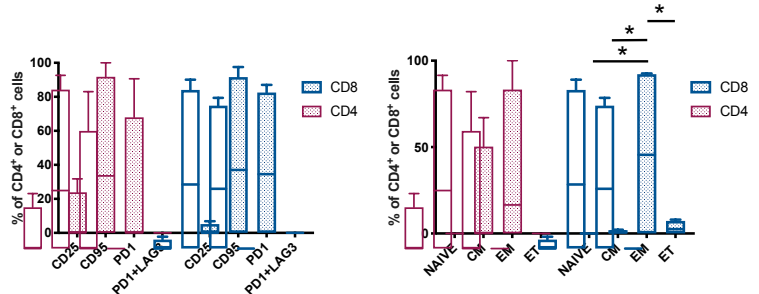

E

H\&E

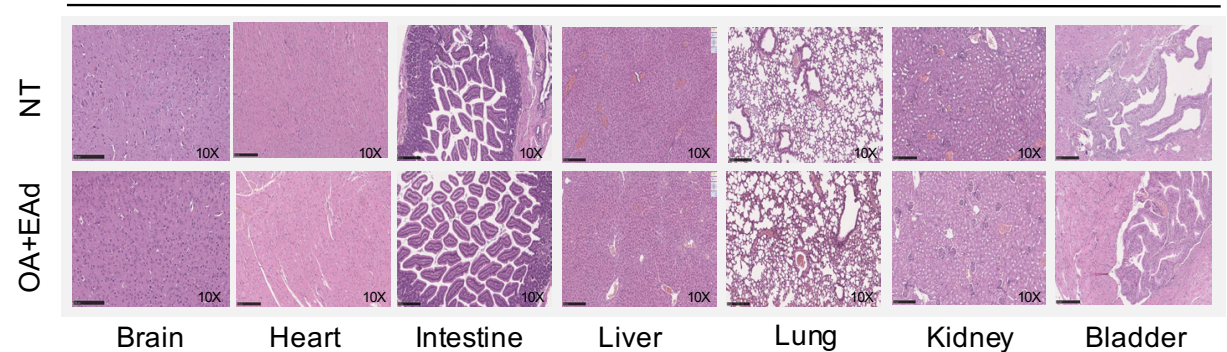

Figure 6 The combinatorial approach shows eradication of the orthotopic tumor, with strong T-cell activation and persistence in vivo and no evidence of toxicity. (A) Analysis of brain sections of two representative mice, in the orthotopic model, by H\&E (left panels) and Ki-67 (right panels) staining. (B) Evaluation of the percentage of circulating human CD45 ${ }^{+}$cells in the peripheral blood (left graph) and analysis of the activation and exhaustion profiles of CD4 ${ }^{+}$and CD8 ${ }^{+}$subpopulations (right graph), analyzed by FC, 40 days and (C) 60 days after treatment. Data are summarized as mean \pm SEM. (D) Evaluation of the percentage of tumor-infiltrating human $\mathrm{CD} 45^{+}$cells in explanted tumor masses (left graph) and analysis of the activation/exhaustion and memory profiles of $\mathrm{CD}^{+}$and $\mathrm{CD}^{+}$subpopulations in the $\mathrm{OA}+\mathrm{EAd}$ treatment group (right graphs), analyzed by $\mathrm{FC}$; (E) representative pictures of the H\&E staining of several, non-target, mouse tissues in the OA+EAd and NT group. EAd, EphA2 adenovirus; NT, untreated; OA, oncolytic adenovirus.

Similarly to what has been reported for adult HGG, we confirmed the relevance of EphA2 as a glioma-associated antigen also in pHGGs, as shown by the correlation between a high expression and worse clinical outcome of patients. ${ }^{20} 25$ In a translational perspective, targeting of EphA2 appears to be safe since the epitope is more accessible during the division of malignant and not on normal cells, ${ }^{26}$ becoming a good candidate target for 
immunotherapies. ${ }^{23}$ 27-29 The rationale for combining an OA with an adenoviral vector is based on the need to overcome the main limitations of each individual platform. As shown in the combinatorial approach, OA allows a significant amplification of the transgene by providing EAd with the required replication machinery. Armed OAs carrying a therapeutic transgene have also been developed; however, we believe that separating oncolysis and gene therapy by the administration of two viral platforms has several advantages. ${ }^{30-32}$ First, the two viruses express different fibers, exploiting separate ligands for cell entry (CAR and integrins for the OA, CD46 for the EAd), thus allowing broader cell targeting. In the extremely heterogenous mass composing solid tumors, and in particular HGGs, cells which are resistant to infection by the OA can instead be infected by EAd and serve as producer of the BiTE. At the same time, separating the two killing mechanisms (oncolysis and T cell-mediated cytotoxicity) reduces the risk of off-tumor treatment toxicity since a simultaneous coinfection of non-target tissue is less likely. On the contrary, the combination treatment induces high intratumoral amplification of the BiTE, thanks to the selective replication of $\mathrm{OA}$ by tumor cells. This leads to engagement and activation of $\mathrm{T}$ cells at the tumor site, overcoming the short half-life of BiTE and the limitations to reach the tumor opposed by the blood-brain barrier and minimizing systemic exposure, therefore increasing the antitumor potency of the treatment and reducing, at the same time, the risk for toxicity.

Fast adenoviral clearance by the host immunity was shown to hamper the oncolytic activity, ${ }^{33}$ owing to the large spread of Ad infections in the community. Thanks to the introduction of a BiTE redirecting the cytotoxic $\mathrm{T}$ lymphocytes (CTLs) against the tumor, we turn this obstacle to our advantage, as recently shown by other groups using armed OAs. ${ }^{32} 34{ }^{35}$ We clearly demonstrate that the engagement of CTLs and tumor cells via BiTE is able to enhance the killing of cancer cells by activation of the cytotoxic potential of $\mathrm{T}$ cells, inducing the death of a proportion of cancer cells that resulted resistant to the oncolytic activity of OA (such as the U87 cell line or the primary pHGG cells). However, we still observed a suboptimal tumor control in the more resistant settings and especially in primary pHGG cells. Therefore, we postulated that the activation of $\mathrm{T}$ cells through CD3 alone could not be sufficient to induce effective antitumor activity, especially in a suppressive tumor environment. This is especially relevant in a perspective of translation to the clinic, since it is well known that (1) the fitness of the immune system of these patients is largely impaired by the conventional treatments, and (2) T-cell activation and persistence are crucial to guarantee long-term tumor control. ${ }^{3637}$ Indeed, Fajardo et aldemonstrated that $\mathrm{T}$ cells persist at tumor sites for 9 days only, in an immunocompromised mouse model that is unable to fully reproduce the suppressive mechanisms occurring at the tumor, thus probably even overestimating the real T-cell persistence in situ. ${ }^{32}$ In 2017 , Velasquez et al validated the importance of costimulation molecules, CD28 or 41BB, to improve the antitumor effector function and persistence of T cells engaged by CD19-BiTE for the clinical treatment of B-cell malignancies. ${ }^{38}$ We therefore decided to include a costimulatory signal in the system, using as proof of concept a human CD28 mAb, due to the well-known importance of this costimulating molecule to drive naive T-cell proliferation and to sustain, as secondary signal, T-cell activation following TCR engagement. ${ }^{39}$

In our approach, the introduction of $\alpha \mathrm{CD} 28$ was able to promote a significant increase in the killing of those tumor cells that had shown a strong resistance to the treatment, including primary pHGG cells. Moreover, $\alpha \mathrm{CD} 28$ significantly increases the activation and proliferation of T cells, inducing only a mild exhaustion, not only in vitro but also in a xenograft in vivo model, with activated $\mathrm{T}$ cells circulating up to 60 days after infusion in the animals treated with the combined OA+EAd. Notably, costimulation does not induce a T-cell terminal differentiation, maintaining a high proportion of CM and EM cells that predict the persistence of CTL activity. The synapse created by the BiTE plays a fundamental role in our strategy, and the introduction of the costimulatory molecule optimizes the lymphocytic activity, as confirmed also at a molecular level by the T-cell gene-expression analysis. It has been recently shown that the generation of T-cell responses against both virus and tumor is more important for the efficacy of oncolytic viruses than direct oncolysis ${ }^{40}$; therefore, we believe that proper and persistent enhancement of this response can significantly improve the outcome of patients.

In order to fully characterize the contribution of oncolytic therapy, the effect of Ad encoding an EphA2BiTE, their combination and the possible additive effect of a costimulatory molecule such as $\alpha \mathrm{CD} 28$, we investigated a gene-expression profile, which clearly underlined the benefit of using a combination therapy such as $\mathrm{OA}+\mathrm{EAd}$ and the importance of the integration of a costimulatory molecule to improve the quality and magnitude of the anti-tumor response of T cells. This effect is most prominent in genes involved in (1) inflammation and activation pathways of $\mathrm{T}$ lymphocytes and in the activation of apoptosis; (2) cell stress, significantly upregulated in tumor cells; (3) in the amelioration of tumor microenvironment-reducing immunosuppressive molecules, such as cytokines and interleukins, in tumor cells.

The results obtained in vitro were further validated in vivo: in both experimental animal models, subcutaneous and more challenging and relevant orthotopic models, we demonstrated an earlier, stronger and long-lasting tumor control on combination of oncolysis and T cellmediated cytotoxic activity. This more sustained tumor control translates into a significantly improved OS of the combined treatment group, at least in the subcutaneous model.

From the perspective of a clinical translation, we envision as a future development of the approach the introduction of a stable costimulatory signal in our system. Indeed, 
the systemic use of the CD28 superagonist TGN1412 has been associated with the development of unacceptable toxicity, namely, multiorgan failure associated to severe cytokine release storm, in all the patients treated in a first-in-human clinical trial in $2006{ }^{41}$ The introduction of an armed OA encoding for a costimulatory signal would enable the intratumor production of the costimulatory molecule, therefore reducing the systemic exposure and improving the safety of the approach. It must be noted, however, that the development of the new CD28 superagonist TAB08 and the administration at consistently lower doses than the first trial (ie, 1000-fold lower) appear to be safe, paving the way for the reintroduction of the systemic use of transient CD28 mAbs in the clinic. ${ }^{42}$ Moreover, we acknowledge that the use of immunodeficient mouse models represents a limitation since it cannot reproduce the human innate antiviral response and the immunological memory.

In conclusion, we demonstrated that the use of an approach combining the oncolytic activity, the T-cell engagement and T-cell costimulation is able to induce significant, long-lasting tumor control in highly aggressive pHGG models. We also provided clear evidence supporting the importance of optimizing the approach with a costimulation, in order to persistently activate host, tumor-redirected $\mathrm{T}$ cells, in view of their fundamental role in the tumor eradication through their inflammatory and cytotoxic antitumor activity.

\author{
Author affiliations \\ ${ }^{1}$ Department of Paediatric Haematology and Oncology, Cell and Gene Therapy, \\ Bambino Gesù Children's Hospital, IRCCS, Rome, Italy \\ ${ }^{2}$ Neurosurgery Unit, Department of Neuroscience and Neurorehabilitation, Bambino \\ Gesù Children's Hospital, IRCCS, Rome, Italy \\ ${ }^{3}$ Pathology Unit, Department of Laboratories, Bambino Gesù Children's Hospital, \\ IRCCS, Rome, Italy \\ ${ }^{4}$ The FIRC Institute of Molecular Oncology, IFOM, Milano, Italy \\ ${ }^{5}$ Institute of Molecular Genetics National Research Council, Pavia, Italy \\ ${ }^{6}$ Flow Cytometry and Histology Core Facilities, Bambino Gesù Children's Hospital, \\ IRCCS, Rome, Italy \\ ${ }^{7}$ Baylor College of Medicine Center for Cell and Gene Therapy, Houston, Texas, USA \\ ${ }^{8}$ Department of Bone Marrow Transplantation and Cellular Therapy, St Jude \\ Children's Research Hospital, Memphis, Tennessee, USA \\ ${ }^{9}$ Department of Pediatrics, Sapienza University of Rome, Roma, Italy
}

Correction notice This paper has been updated since first published to amend author name 'Lucia Lisa Petrilli'.

Acknowledgements The authors thank Professor Cerullo for kindly providing the Ad5 $\Delta 24$ and Dr Algeri and Dr Tomao for providing mesenchymal stromal cells derived from healthy donors (HDs). The authors acknowledge all the donors, both HDs and patients with their families, for providing essential support for their research.

Contributors FDB and IC share last authorship. FDB, IC and FL designed experimental studies, supervised the project, analyzed the data and wrote the manuscript. CMA, GW and LA performed the in vitro and in vivo experiments. VAP led the setup of the animal models and performed the in vivo experiments. AC and AM provided the patient samples and their expertise to define the clinical translation perspective. FDC provided the neuropathology expert evaluation. PL helped in establishing and characterizing the primary pediatric high-grade glioma (pHGG) cell lines, and MV provided her expertise on PHGG characterization. IC, FF and ES performed the bioinformatic analysis of the gene-expression data. MS and CDS performed, respectively, the flow-cytometry acquisition and the preparation of the pathology specimen and stainings. MKB and SG provided their expertise for the EAd cloning. CQ, MKB, SG, BDA and FL also provided expertise in the immunotherapy field and critically reviewed the manuscript.

Funding This work was partly supported by grants from AIRC (Associazione Italiana Ricerca sul Cancro, My First AIRC, ID 20450, to FDB; investigator grant, ID 21724, and Special Program Metastatic disease: the key unmet need in oncology 5 per mille 2018, ID 21147, to FL; start-up grant, ID 17184, to IC), Ministero della Salute (Ricerca Corrente to FDB and IC; Ricerca Corrente-Progetto CAR T, ID RCR-2019-23669115 to FL; Ricerca Finalizzata, ID RF-2016-02364388, to FL), Ministero dell'Istruzione, Università e Ricerca (MIUR, Project PRIN 2017, ID 2017WC8499, to FL).

Competing interests None declared.

Patient consent for publication Not required.

Ethics approval Both parents and healthy donors signed a written informed consent, in accordance with rules set by the institutional review board of Bambino Gesù Children Hospital, IRCCS, Rome, Italy (OPBG; Approval of Ethical Committee $N^{\circ} 969 / 2015$ prot. $N^{\circ} 669 L B$, and $N^{\circ} 1422 / 2017$ prot. №810). In vivo experiments were performed in accordance with national and international ethical requirements and were approved by the Italian Health Ministry ( $\left.\mathrm{N}^{\circ} 88 / 2016-\mathrm{PR}\right)$.

Provenance and peer review Not commissioned; externally peer reviewed.

Data availability statement Data are available upon reasonable request.

Supplemental material This content has been supplied by the author(s). It has not been vetted by BMJ Publishing Group Limited (BMJ) and may not have been peer-reviewed. Any opinions or recommendations discussed are solely those of the author(s) and are not endorsed by BMJ. BMJ disclaims all liability and responsibility arising from any reliance placed on the content. Where the content includes any translated material, BMJ does not warrant the accuracy and reliability of the translations (including but not limited to local regulations, clinical guidelines, terminology, drug names and drug dosages), and is not responsible for any error and/or omissions arising from translation and adaptation or otherwise.

Open access This is an open access article distributed in accordance with the Creative Commons Attribution Non Commercial (CC BY-NC 4.0) license, which permits others to distribute, remix, adapt, build upon this work non-commercially, and license their derivative works on different terms, provided the original work is properly cited, appropriate credit is given, any changes made indicated, and the use is non-commercial. See http://creativecommons.org/licenses/by-nc/4.0/.

\section{ORCID iDs}

Gerrit Weber http://orcid.org/0000-0001-7240-0300

Concetta Quintarelli http://orcid.org/0000-0002-4343-9705

Stephen Gottschalk http://orcid.org/0000-0003-3991-7468

Valentina Hoyos http://orcid.org/0000-0002-8269-6341

Ignazio Caruana http://orcid.org/0000-0002-9250-0605

Francesca Del Bufalo http://orcid.org/0000-0001-9643-3465

\section{REFERENCES}

1 Ostrom QT, Gittleman H, Liao P, et al. CBTRUS statistical report: primary brain and central nervous system tumors diagnosed in the United States in 2007-2011. Neuro Oncol 2014;16:iv1-63.

2 Kieran MW, Hargrave DR, Cohen KJ, et al. Phase 1 study of dabrafenib in pediatric patients (pts) with relapsed or refractory $B R A F$ V600E high- and low-grade gliomas (HGG, LGG), Langerhans cell histiocytosis (LCH), and other solid tumors (OST). JCO 2015;33:10004-04.

3 Liang M-L, Ma J, Ho M, et al. Tyrosine kinase expression in pediatric high grade astrocytoma. J Neurooncol 2008;87:247-53.

4 Chuntova P, Downey KM, Hegde B, et al. Genetically engineered T-cells for malignant glioma: overcoming the barriers to effective immunotherapy. Front Immunol 2018;9:3062.

5 Twumasi-Boateng K, Pettigrew JL, Kwok YYE, et al. Oncolytic viruses as engineering platforms for combination immunotherapy. Nat Rev Cancer 2018;18:419-32.

6 Sayedahmed EE, Kumari R, Mittal SK. Current use of adenovirus vectors and their production methods. Methods Mol Biol 2019;1937:155-75.

7 Chiocca EA, Rabkin SD. Oncolytic viruses and their application to cancer immunotherapy. Cancer Immunol Res 2014;2:295-300.

8 Parato KA, Senger D, Forsyth PAJ, et al. Recent progress in the battle between oncolytic viruses and tumours. Nat Rev Cancer 2005;5:965-76. 
9 Martínez-Vélez N, Garcia-Moure M, Marigil M, et al. The oncolytic virus Delta-24-RGD elicits an antitumor effect in pediatric glioma and DIPG mouse models. Nat Commun 2019;10:2235.

10 Garcia-Carbonero R, Salazar R, Duran I, et al. Phase 1 study of intravenous administration of the chimeric adenovirus enadenotucirev in patients undergoing primary tumor resection. $J$ Immunother Cancer 2017:5:71.

11 Ranki T, Pesonen S, Hemminki A, et al. Phase I study with ONCOS-102 for the treatment of solid tumors - an evaluation of clinical response and exploratory analyses of immune markers. $J$ Immunother Cancer 2016;4:17.

12 Eissa IR, Bustos-Villalobos I, Ichinose T, et al. The current status and future prospects of oncolytic viruses in clinical trials against melanoma, glioma, pancreatic, and breast cancers. Cancers 2018;10. doi:10.3390/cancers10100356. [Epub ahead of print: 26 Sept 2018].

13 Wang Y, Zhou X, Wu Z, et al. Preclinical safety evaluation of oncolytic herpes simplex virus type 2. Hum Gene Ther 2019;30:651-60.

14 Reid T, Warren R, Kirn D. Intravascular adenoviral agents in cancer patients: lessons from clinical trials. Cancer Gene Ther 2002;9:979-86.

15 Diaconu I, Cerullo V, Hirvinen MLM, et al. Immune response is an important aspect of the antitumor effect produced by a CD40Lencoding oncolytic adenovirus. Cancer Res 2012;72:2327-38.

16 Bernt KM, Ni S, Tieu A-T, et al. Assessment of a combined, adenovirus-mediated oncolytic and immunostimulatory tumo therapy. Cancer Res 2005;65:4343-52.

17 Hoyos V, Del Bufalo F, Yagyu S, et al. Mesenchymal stromal cells for linked delivery of oncolytic and apoptotic adenoviruses to non-smallcell lung cancers. Mol Ther 2015;23:1497-506.

18 Haas C, Krinner E, Brischwein K, et al. Mode of cytotoxic action of T cell-engaging bite antibody MT110. Immunobiology 2009;214:441-53.

19 Nagorsen D, Baeuerle PA. Immunomodulatory therapy of cancer with T cell-engaging bite antibody blinatumomab. Exp Cell Res 2011;317:1255-60.

20 Wang L-F, Fokas E, Bieker M, et al. Increased expression of EphA2 correlates with adverse outcome in primary and recurrent glioblastoma multiforme patients. Oncol Rep 2008;19:151-6.

21 von Stackelberg A, Locatelli F, Zugmaier G, et al. Phase I/Phase II study of Blinatumomab in pediatric patients with relapsed/refractory acute lymphoblastic leukemia. J Clin Oncol 2016;34:4381-9.

22 Chow KKH, Naik S, Kakarla S, et al. T cells redirected to EphA2 for the immunotherapy of glioblastoma. Mol Ther 2013;21:629-37.

23 Iwahori K, Kakarla S, Velasquez MP, et al. Engager T cells: a new class of antigen-specific T cells that redirect bystander T cells. Mol Ther 2015;23:171-8.

24 Yu F, Wang X, Guo ZS, et al. T-cell engager-armed oncolytic vaccinia virus significantly enhances antitumor therapy. Mol Ther 2014:22:102-11.

25 Hogarth RM, Gibbs BJ, McKenzie CR, et al. Learning from feedback: exactingness and incentives. J Exp Psychol Learn Mem Cogn 1991:17:734-52.

26 Coffman KT, Hu M, Carles-Kinch K, et al. Differential EphA2 epitope display on normal versus malignant cells. Cancer Res 2003:63:7907-12.
27 Bielamowicz K, Fousek K, Byrd TT, et al. Trivalent CAR T cells overcome interpatient antigenic variability in glioblastoma. Neuro Oncol 2018;20:506-18.

28 Pollack IF, Jakacki RI, Butterfield LH, et al. Antigen-specific immunoreactivity and clinical outcome following vaccination with glioma-associated antigen peptides in children with recurrent high-grade gliomas: results of a pilot study. J Neurooncol 2016;130:517-27.

29 Shitara K, Satoh T, Iwasa S, et al. Safety, tolerability, pharmacokinetics, and pharmacodynamics of the afucosylated, humanized anti-EPHA2 antibody DS-8895a: a first-in-human phase I dose escalation and dose expansion study in patients with advanced solid tumors. J Immunother Cancer 2019;7:219.

30 Burke JM, Lamm DL, Meng MV, et al. A first in human phase 1 study of CG0070, a GM-CSF expressing oncolytic adenovirus, for the treatment of nonmuscle invasive bladder cancer. J Urol 2012;188:2391-7.

31 Heiniö C, Havunen R, Santos J, et al. TNFa and IL2 encoding oncolytic adenovirus activates pathogen and danger-associated immunological signaling. Cells 2020;9. doi:10.3390/cells9040798. [Epub ahead of print: 26 Apr 2020].

32 Fajardo CA, Guedan S, Rojas LA, et al. Oncolytic adenoviral delivery of an EGFR-Targeting T-cell Engager improves antitumor efficacy. Cancer Res 2017;77:2052-63.

33 Ferguson MS, Lemoine NR, Wang Y. Systemic delivery of oncolytic viruses: hopes and hurdles. Adv Virol 2012;2012:1-14.

34 Freedman JD, Duffy MR, Lei-Rossmann J, et al. An oncolytic virus expressing a T-cell Engager simultaneously targets cancer and immunosuppressive stromal cells. Cancer Res 2018;78:6852-65.

35 Rosewell Shaw A, Porter CE, Watanabe N, et al. Adenovirotherapy delivering cytokine and checkpoint inhibitor augments CAR $T$ cells against metastatic head and neck cancer. Mol Ther 2017;25:2440-51.

36 Caruana I, Weber G, Ballard BC, et al. K562-derived wholecell vaccine enhances antitumor responses of CAR-redirected virus-specific cytotoxic T lymphocytes In Vivo. Clin Cancer Res 2015;21:2952-62.

37 Jafarzadeh L, Masoumi E, Fallah-Mehrjardi K, et al. Prolonged persistence of chimeric antigen receptor (CAR) T cell in adoptive cancer immunotherapy: challenges and ways forward. Front Immunol 2020;11:702.

38 Velasquez MP, Szoor A, Vaidya A, et al. Cd28 and 41BB costimulation enhances the effector function of CD19-specific Engager T cells. Cancer Immunol Res 2017;5:860-70.

39 Esensten JH, Helou YA, Chopra G, et al. Cd28 costimulation: from mechanism to therapy. Immunity 2016;44:973-88.

$40 \mathrm{Li} \mathrm{X}$, Wang P, Li H, et al. The efficacy of oncolytic adenovirus is mediated by T-cell responses against virus and tumor in Syrian hamster model. Clin Cancer Res 2017;23:239-49.

41 Suntharalingam G, Perry MR, Ward S, et al. Cytokine storm in a phase 1 trial of the anti-CD28 monoclonal antibody TGN1412. N Engl J Med 2006;355:1018-28.

42 Tyrsin D, Chuvpilo S, Matskevich A, et al. From TGN1412 to TAB08: the return of $\mathrm{CD} 28$ superagonist therapy to clinical development for the treatment of rheumatoid arthritis. Clin Exp Rheumatol 2016;34:45-8. 


\section{Correction: Oncolytic adenovirus and gene therapy with EphA2-BiTE for the treatment of pediatric high-grade gliomas}

Arnone CM, Polito VA, Mastronuzzi A, et al. Oncolytic adenovirus and gene therapy with EphA2-BiTE for the treatment of pediatric high-grade gliomas. J Immunother Cancer 2021;9:e001930. doi: 10.1136/jitc-2020-001930

This paper has been updated since first published to amend author name 'Lucia Lisa Petrilli'.

Open access This is an open access article distributed in accordance with the Creative Commons Attribution Non Commercial (CC BY-NC 4.0) license, which permits others to distribute, remix, adapt, build upon this work non-commercially, and license their derivative works on different terms, provided the original work is properly cited, appropriate credit is given, any changes made indicated, and the use is non-commercial. See http://creativecommons.org/licenses/by-nc/4.0/.

(c) Author(s) (or their employer(s)) 2021. Re-use permitted under CC BY-NC. No commercial re-use. See rights and permissions. Published by BMJ.

J Immunother Cancer 2021;9:e001930corr1 . doi:10.1136/jitc-2020-001930corr1 